\title{
Critical Temperature and Nonextensivity in Long-range Interacting Lennard-Jones-like Fluids
}

\author{
Sergio Curilef ${ }^{1,2}$ and Constantino Tsallis ${ }^{2,3}$ \\ 1 Departamento de Física, Universidad Católica del Norte, Casilla 1280, Av. Angamos 0610, \\ Antofagasta, Chile \\ ${ }^{2}$ Centro Brasileiro de Pesquisas Físicas, Rua Xavier Sigaud 150, 22290-180 Rio de Janeiro-RJ, \\ Brazil \\ 3 Niels Bohr Institute, Blegdamsvej 17, DK-2100 Copenhagen, Denmark
}

\begin{abstract}
Molecular dynamic simulations for systems with $D=2,3$ Lennard-Jones-like interactions are studied. In the model, we assume that, at long distances, the two-body attractive potential decays as $r^{-\alpha}$. Thermodynamic extensivity (nonextensivity) is observed for $\alpha>D(0 \leq \alpha \leq D)$. Particular attention is payed to the liquid-gas critical point located, in the temperature-pressure plane, at $\left(T_{c}, P_{c}\right) .\left(T_{c}, P_{c}\right)$ are, in the $N \rightarrow \infty$ limit ( $N \equiv$ number of molecules), finite for $\alpha>D$ and diverge for $\alpha \leq D\left(\right.$ as $(\alpha-D)^{-1}$ for $\left.\alpha / D \rightarrow 1+0\right)$. However, the variables $T_{c}^{*} \equiv T_{c} / N^{*}$ and $P_{c}^{*} \equiv P_{c} / N^{*}$ with $N^{*} \equiv\left[N^{1-\alpha / D}-1\right] /[1-\alpha / D]$ remain finite for all $\alpha$. Thus, the extensive and nonextensive regions become unified, as recently conjectured. These results should be useful for discussing gravitation and some special fluids.
\end{abstract}

PACS numbers: 05.70.Ce, 05.70.Fh, 64.90.+b

In recent years, much attention has been paid to physical systems with microscopic longrange interactions. Nonextensive behavior and difficulties with the standard formulation of thermodynamics are since long known for this class of systems (see, for instance, [1,2]). More precisely, in order to have a well defined thermodynamic limit in the usual sense, a system must have a finite free energy per particle. In other words, an increase of $N$ (number of particles) is expected to leave the free energy per particle unaltered if $N>>1$, i.e., the free energy $F_{N}$ should be asymptotically proportional to $N$. However, when the effective range of the interactions between particles decays slowly enough with the distance, $F_{N}$ increases with a higher power of $N$, hence $F_{N} / N$ diverges when $N \rightarrow \infty$. According to many text-book interpretations of thermodynamics, the concept itself of thermodynamic limit is ill-defined in such cases and the formalism becomes unphysical (see, for instance, [1]). In some sense (that will become transparent later on), this is a restricted interpretation of thermodynamics and a possible way out from this difficulty has been recently proposed [3] and illustrated through several examples [2, 1.5 . In the present effort, we shall address along these lines a Lennard-Jones-like fluid, with special emphasis onto its gas-liquid phase transition and the associated critical point. To do this we shall perform molecular dynamics for the two- and three-dimensional cases.

In order to understand the thermodynamical behavior of systems with long-range interacting particles, we consider a Lennard-Jones-like model with pair interactions characterized by a $(12, \alpha)$ potential. A variety of values for $\alpha$ can be associated with standard interactions in models for fluids. For instance, for $D=3, \alpha=6$ corresponds to the standard Lennard-Jones fluid, $\alpha=3$ essentially corresponds to dipole-dipole interactions in systems with dipole configurations such that the interaction is attractive, $\alpha=2$ corresponds to 
dipole-monopole interactions (such us those relevant in the tides), $\alpha=1$ mimics the gravitational and the (unscreened) Coulombic interactions, and finally $\alpha=0$ corresponds to a mean field approach. For arbitrary $D$, the choice $\alpha=D-2$ corresponds to the isotropic solutions of the $D$-dimensional Poisson equation. Also, there are some special situations in which neutral, glassy, small spheres can interact through long-range potentials with low, and not even integer, $\alpha$. Such cases might occur, through a Casimir-like effect due to the large fluctuations at the critical point of standard fluids, within which the little spheres are immersed [6]. We might occasionally refer to such fluids as Casimir-like ones. For all these reasons, we shall focus on herein all the values of $\alpha$ in the interval $0 \leq \alpha \leq 6$.

An interesting though preliminary discussion of this class of fluids has been done by Grigera [4]. Our aim in the present work is to provide a more detailed description which will hopefully give a suitable picture of some general thermodynamic properties of systems with long-range (and not singular at the origin) interactions.

The two-body potential we shall assume is given as follows:

$$
v(r)=4 \epsilon\left[\left(\frac{\sigma}{r}\right)^{12}-\frac{1}{4} \frac{(48 / \alpha)^{\alpha / 12}}{(1-\alpha / 12)^{1-\alpha / 12}}\left(\frac{\sigma}{r}\right)^{\alpha}\right],
$$

where $r$ is the distance between particles and $\sigma>0$ and $\epsilon>0$ are specific Lennard-Jones-like parameters. For the long-tailed attractive term $r^{-\alpha}$ in potential (1) we consider $0 \leq \alpha<12$. The $r^{-12}$ term describes the short range repulsive potential (due to nonbonding overlap between the electron clouds for the $D=3$ case). The usual convention in the theory of fluids (for instance, standard Lennard-Jones fluids) is to define the diameter $\sigma$, via $v(\sigma)=0$. Due to the range of the interactions, this would not seem particularly helpful here. So, we rather make a different convention, namely the $r^{-\alpha}$ coefficient has been chosen so that the minimal value $v\left(r_{\min }\right)=\epsilon$ in all cases. The Lennard-Jones-like $(12, \alpha)$ potentials as a function of $r$ are plotted in FIG 11 for typical values of $\alpha$.

A main difficulty for fluid systems with long-range (but not infinitely long) interactions is the absence of any exact solution. In our present case, calculations were performed with the molecular dynamics method for systems of $N$ spherically-symmetric particles with periodic boundary conditions. Standard mean field (or van der Waals) theory for an integrable (i.e., $\alpha>D)$ potential with a cutoff $r_{c}$ uses corrections $\propto \int_{r_{c}}^{\infty} v(r) d^{D} r$. However, our main aim is to discuss the trend of thermodynamic quantities as a function of $N$ (the size of the system) and to provide an unified approach to the thermodynamic limit for the entire range $(0 \leq \alpha \leq 6)$. Consequently, no corrections are considered in the present treatment and a cutoff distance of half of the box size was applied to the interaction; we verified that, in the $N \rightarrow \infty$ limit, no physical consequences seemed to emerge from the adoption of this computational convenience. The equations of motion were solved using the Verlet algorithm [7] and the temperature was kept constant by a weak coupling of the system with an external thermal bath [8].

When starting a simulation from scratch, the initial configuration is generated by randomly distributing the molecules. Then, the molecules evolve in such a way as to achieve the energetically most favorable positions and velocities. Full equilibrium is assumed only when the pressure, the potential energy and the total energy exhibit stable values. In general, the computational work is relatively heavy, since for systems with long-range interactions it is uneasy to obtain very precise numerical data for quantities such as the critical temperature, 
for example. Nevertheless, as we will see, the trends of the present numerical data clearly exhibit, in all the cases we have studied here, the conjectured thermodynamical scalings [3].

In the remainder of this paper, quantities are specified in usual reduced (dimensionless) form in terms of the Lennard-Jones parameters $\sigma$ and $\epsilon$. The reduced number density is $\rho \sigma^{D}$ where $\rho=N / V$; the reduced temperature is $k_{B} T / \epsilon$ where $k_{B}$ is the Boltzmann constant; the reduced pressure is $\sigma^{D} P / \epsilon$; finally, the reduced time is $\sqrt{\epsilon / m} t / \sigma$, where $m$ is the mass of the particles. From now on we consider $\sigma=\epsilon=m=k_{B}=1$.

Special attention is payed to the point at which the critical isotherm $\left(T=T_{c}\right.$, where $T_{c}$ corresponds to the critical temperature) simultaneous has a vanishing slope $(\partial P / \partial V)_{T_{c}}=$ 0 and an inflection point, i.e., where the curve changes from convex to concave, hence, $\left(\partial^{2} P / \partial V^{2}\right)_{T_{c}}=0$ in the pressure-volume plane. In fact, these properties simultaneously occur only at the critical point of the system. It is known that the standard LennardJones fluid provides [9] a handy model for testing liquid theories and for investigating such phenomena as melting, the liquid-vapour surface, nucleation, etc. Consistently, our interest in the present more general fluid stems mainly in the critical point of the liquid-vapor curve.

The NVT-ensemble was used in our simulations. Naturally, strictly speaking, only at the thermodynamical limit we can speak of the critical temperature. Nevertheless, even for finite $N$, we can define an effective "critical" temperature $T_{c}(\alpha ; N)$ by using the zero slope and inflection point criteria just mentioned. We have obtained the values for $T_{c}(\alpha ; N)$ from simulations with systems containing $N=27,64,125,216$ and 512 in three dimensions, and $N=25,49,100,225$ and 400 in two dimensions.

In FIG 2, $T_{c}(\alpha ; N)$ as a function of $N$ is depicted for typical values of $\alpha$ for $\mathrm{D}=3$ case. For $\alpha>D$, the critical temperature shows a tendency to saturate in the limit $N \rightarrow \infty$, but the convergence becomes very slow when $\alpha$ approaches $D$ from above, consistently with what was already discussed for the total and the potential energies of a similar fluid [4]; as we shall see, for $\alpha \leq D$, the approximate critical temperature diverges with $N$. A suitable plot for the curves associated with the extensive regime (i.e., $\alpha>D$ ) is depicted at inset in FIG 2. Labels on lines correspond to several values of $\alpha$ at Figure $(\alpha=1,2,3,4,5,6)$ and inset $(\alpha=3.5,4,5,6)$. The $D=2$ case exhibits similar trends. In fact, all our results (for all $(\alpha, D))$ are satisfactorily fitted with the simple expression $T_{c}(\alpha ; N) \approx T_{c}(\alpha)+a_{\alpha} N^{1-\alpha / D}$, where the parameters $T_{c}(\alpha)$ and $a_{\alpha}$ come out from the fitting. For the extensive region $(\alpha>D) T_{c}(\alpha)$ represents the thermodynamic limit of the critical temperature; $a_{\alpha}<0$.

In FIG 3, we depict some values of $T_{c}(\alpha)$ given by the present approach as a function of $\alpha$. We verify that $T_{c}$ diverges for $\alpha \leq D$ and that, when $\alpha \rightarrow D$ from above, $T_{c}(\alpha)$ diverges like $1 /(\alpha-D)$ (see the inset in the figure). This behavior has been conjectured [3] for generic systems with long-range interactions. The thermodynamic limit is not reached in the usual way in the nonextensive region. Nevertheless, if we appropriately scale the various relevant quantities, our numerical results will show that this limit is in fact as well defined as that corresponding to the extensive region. Let us now clarify how this happens.

Thermodynamic quantities $\mathcal{A}_{N}$ like the internal energy, the free energy, Gibbs energy, etc., associated with systems including potentials having an attractive tail that decays as $r^{-\alpha}$, do not always scale with $N$. Indeed, we generically have

$$
\frac{\mathcal{A}_{N}}{N} \propto \int_{1}^{N^{1 / D}} d r \frac{r^{D-1}}{r^{\alpha}}=\frac{1}{D} \frac{N^{1-\alpha / D}-1}{1-\alpha / D}
$$


Consistently, we can introduce [2, 3] a new variable given by

$$
N^{*} \equiv \frac{N^{1-\alpha / D}-1}{1-\alpha / D}
$$

which, in the limit $N \rightarrow \infty$, behaves asymptotically as $1 /(\alpha / D-1)$ for $\alpha / D>1$; as $\ln N$ for $\alpha / D=1$; and as $N^{1-\alpha / D} /(1-\alpha / D)$ for $0 \leq \alpha / D<1$. For $\alpha=0, N^{*} \sim N$, which precisely recovers the value frequently used in mean field approximations in order to renormalize the coupling constants in such a way as to make the system to (artificially) become extensive. It is known since several decades ( [10] and references therein) that thermodynamical extensivity imposes, in classical systems like the present one, $\alpha / D>1$. Our definition (3) clearly is consistent with this, but also provides the correct scaling for $0 \leq \alpha / D \leq 1$, where no essential knowledge has been developed until very recently, and along the present lines.

We expect the thermodynamic variables to generically include scaling with $N^{*}$ as recently conjectured [3]. For instance, we expect the $\lim _{N \rightarrow \infty} \mathcal{A}_{N} /\left(N N^{*}\right)$ to be finite for all values of $\alpha$, whether it is in the extensive region or in the nonextensive one. Thus, we can write

$$
\frac{G_{N}}{N^{*} N}=\frac{U_{N}}{N^{*} N}-\frac{T}{N^{*}} \frac{S_{N}}{N}+\frac{P}{N^{*}} \frac{V_{N}}{N} .
$$

It can be easily verified that this type of scaling preserves the Legendre transformation structure of thermodynamics, even in the nonextensive region $(0 \leq \alpha \leq D)$ [3]. These features are in fact included in a more general context, namely that of a recent statisticalmechanical formalism addressing nonextensive systems [11 [13]. In what concerns the so called intensive variables $\left(T, P\right.$, etc), we expect them to scale with $N^{*}$. For instance, the critical temperatures must scale as $T_{c}(\alpha ; N) \sim N^{*} T_{c}^{*}(\alpha)$. Indeed, in FIG $\mathbb{Q}(a)$, the critical temperature $T_{c}(\alpha ; N)$ vs $N^{*}$ is exhibited for typical values of $\alpha$, and a linear dependence with $N^{*}$ is clearly observed. As before, it is possible to fit the data with the simple expression $T_{c}(\alpha ; N) \approx b_{\alpha}+T_{c}^{*}(\alpha) N^{*}$, where $b_{\alpha}<0$ and $T_{c}^{*}(\alpha)$ are fitting parameters. Since $T_{c}^{*}$ is finite in all cases, it is to be considered as the correctly scaled expression for the critical point of the system. In FIG $⿴ 囗 ⿱ 一 一)(b)$, we plot $T_{c}^{*}$ as a function of $\alpha$ in both the extensive and nonextensive regimes. We see that $T_{c}^{*}(\alpha)$ increases continuosly with $\alpha$ but its derivative $d T_{c}^{*}(\alpha) / d \alpha$ possibly presents a discontinuity at $\alpha=D$. The error bars illustrate the mean error (less than 5\%) observed in the measurements of $T_{c}$ in our numerical simulations.

In FIG $5(a)$, we plot $P_{c}(\alpha ; N) / T_{c}(\alpha ; N)$ as a function of $1 / N^{1 / 3}$ for the $D=3$ system, and it turns out to be once again linear. This enables a simple extrapolation (the precise finite size scalings associated with this problem have not been taken into account here, since they do not appear to add anything specially relevant for our present discussion) which provides $P_{c}(\alpha) / T_{c}(\alpha)$, which of course coincides with $P_{c}^{*}(\alpha) / T_{c}^{*}(\alpha)$, since both $P$ and $T$ scale with $N^{*}$. We notice that, as it happened with $T_{c}^{*}(\alpha)$, the values are finite in both the extensive and nonextensive regions (i.e., $\forall \alpha)$ : see FIG $5(b)$. The observed error $(\approx 10 \%$ or less $)$ in the measurement of the $P_{c}$ is roughly the double of that observed for $T_{c}$.

Let us finally address a more general situation, namely the functional relationships such as the equation of states, in the present case the funtion $f\left(P^{*}, \rho, T^{*}\right)=0$, which relates the density $\rho$ with temperature and pressure. In FIG $6(a)$, we illustrate the ideas with the $D=3$ system using several sizes, namely $N=27,64,125,216$ at the same fixed temperature 
$T=3.4$ and $\alpha=2$. We notice, as expected, that the curves in the $(P, \rho)$ plane do not remain finite as $N$ increases. There is no usual thermodynamic limit and consequently no interesting properties can be exhibited under these conditions. The present class of models might produce a negative (here, unphysical) pressure $P_{c}(2 ; N)$ when $N$ increases. Such behavior is commonly observed in $N$-dependent isotherms in the $P-\rho$ plane. However, and interestingly enough, a completely different picture emerges if, instead of presenting the results at fixed $T$, we do it at fixed $T^{*}=T / N^{*}$. Indeed, in FIG $6(b)$, we plot the curves corresponding to $T^{*}=0.43\left(>T_{c}^{*}(2)\right)$ and we observe that the curves start converging as $N$ increases. In fact, the convergence can be exhibited versus $1 / N^{1 / D}$ (see inset), which allows the establishment of a well defined thermodynamic limit even for this example in the nonextensive region. In addition to these results, we appoint that a new family of isotherms is obtained in the present way, when the normalized function $T^{*}(\alpha ; N)$ instead of $T(\alpha ; N)$ is used, but the true temperature value is always given by $T(\alpha ; N)$ in all cases. In the same line, we verify that the values of $P(2 ; N) / T(2 ; N)$ appear to converge, as desired, to nonnegative values when $N \rightarrow \infty$.

At this point, it is important to clarify that the thermodynamical variables we are analyzing are those which appear in relations such as the equation of states. The parameters which characterize thermodynamical equilibrium are the usual ones, i.e., the temperature $T$ of the external thermal bath, its pressure $P$, etc.

Summarizig, we have shown an example, a Lennard-Jones-like fluid, that can violate the usual scalings of thermodynamics, namely those corresponding to extensive systems, i.e., those associated with interactions whose range is not too long, more precisely satisfying $\alpha>$ $D$. We have shown how the appropriate scaling (through $N^{*}$ given by definition (5)) of the various thermodynamical quantities enables an unified picture for both short- and long-range interacting systems. By so doing, we have empirically established a variety of secondary finite-size scalings. In addition to this, we have verified that the critical temperatures, pressures, etc, diverge like $1 /(\alpha-D)$ when $\alpha$ approaches $D$ from above, a fact which is expected to be model-independent. Let us finally add that we hope to have provided enough arguments to show that thermodynamics and the concept of thermodynamical equilibrium can very well accomodate the (until now almost unexplored) nonextensive systems. The detailed study of more such systems (quantum, frustrated, etc) certainly is very welcome.

One of us (S.C.) ackowledges partial support by FONDECYT, grant 3980014. The other one (C.T.) is thankful to Per Bak for useful remarks and warm hospitality at the Niels Bohr Institute where this work was concluded. Finally, CNPq and PRONEX (Brazilian Agencies) are also ackowledged for partial support. 


\section{REFERENCES}

[1] B.J. Hiley and G.S. Joyce, Proc. Phys. Soc., 493 (1965); P.T. Landsberg, J. Stat. Phys. 35, 159 (1984); C. Anteneodo and C. Tsallis, Phys. Rev. Lett. 80,5313 (1998).

[2] P. Jund, S.G. Kim and C. Tsallis, Phys. Rev. B 52, 50 (1995).

[3] C. Tsallis, Fractals 3, 541 (1995).

[4] J.R. Grigera, Phys. Lett. A 217, 47 (1996).

[5] S.A. Cannas and F.A. Tamarit, Phys. Rev. B 54, 12661 (1996); S.A. Cannas and A.C.N. Magalhaes, J. Phys. A 30, 3345 (1997); L.C. Sampaio, M.P. Albuquerque and F.S. de Menezes, Phys. Rev. B 55, 1 (1997).

[6] T.W. Burkhardt and E. Eisenriegler, Phys. Rev. Lett. 74 (1995) 3189.

[7] M.P. Allen and D.J. Tildesley, Computer Simulations in Liquids, (Oxford Science Publications, 1994).

[8] H.J.C. Berendsen, J.P.M. Postma, W.F. van Gunstern, A. DiNola and J.R. Haak, J. Chem. Phys. 81 (1984) 3684.

[9] P. Jetzer, Phys. Rep. 220 (1992) 163.

[10] M.E.Fisher and J.L. Lebowitz, Comm. Math. Phys., 19 (1970) 251.

[11] C. Tsallis, J. Stat. Phys. 52 (1988) 479; an updated Bibliography is available at http://tsallis.cat.cbpf.br/biblio.htm; for a recent review see C. Tsallis, in Nonextensive statistical mechanics and thermodynamics, eds. S.R.A. Salinas and C. Tsallis, Braz. J. Phys. 29, 1 (1999) cond-mat/9903356.

[12] E.M. F. Curado and C. Tsallis, J. Phys. A: Math. Gen 24 (1991) L69; Corrigenda: J. Phys. A:Math. Gen 24 (1991) 3187; 25 (1992) 539.

[13] C. Tsallis, Chaos, Solitons and Fractals 6 (1995) 539. 


\section{FIGURES}

FIG. 1. Lennard-Jones-like $(12, \alpha)$ potentials as functions of $r$ for $\alpha=0,1,2,4,6$.

FIG. 2. Approximate critical temperature $T_{c}(\alpha ; N)$ as a function of $N$ for the $D=3$ system, for $\alpha=1,2,3,4,5,6$. Inset: In the extensive case $(\alpha>D)$, numerical data (circles) were fitted using $T_{c}(\alpha ; N) \approx T_{c}(\alpha)+a_{\alpha} N^{1-\alpha / D}$ (solid line), for $\alpha=3.5,4,5,6$.

FIG. 3. Thermodynamic limit critical temperature $T_{c}(\alpha)$ as a function of $\alpha$ for $D=2,3$. Inset: $1 / T_{c}(\alpha)$ versus $\alpha$. For $\alpha \leq D$ the critical temperature $T_{c}$ diverges.

FIG. 4. (a) The $D=3$ approximate critical temperatures $T_{c}(\alpha ; N)$ as functions of $N^{*}$ for $\alpha=1,2,3$ : Numerical data (circles) and their fitting using $T_{c}(\alpha ; N) \approx b_{\alpha}+T_{c}^{*}(\alpha) N^{*}$ (solid line); (b) Thermodynamic limit for the scaled critical temperature $T_{c}^{*}(\alpha)$ as a function of $\alpha$ for the $D=2,3$ systems.

FIG. 5. (a) The ratio $P_{c}(\alpha ; N) / T_{c}(\alpha ; N)$ versus $1 / N^{1 / 3}$ in both extensive and nonextensive regions for the $D=3$ system; $(b)$ The thermodynamic limit values of $P_{c}(\alpha) / T_{c}(\alpha)$ versus $\alpha$ for the $D=2,3$ systems.

FIG. 6. The ratio $P(\alpha) / T(\alpha)$ versus the density $\rho$ for $\alpha=2$ for the $D=3$ system with $N=27,64,125,216$ particles, at fixed $T=3.4(a)$, and at fixed $T^{*}=0.43(b)$. Inset of $(b)$ : The ratio as a function of $1 / N^{1 / 3}$ for typical values of $\rho$ in order to illustrate their (linear) convergence. 


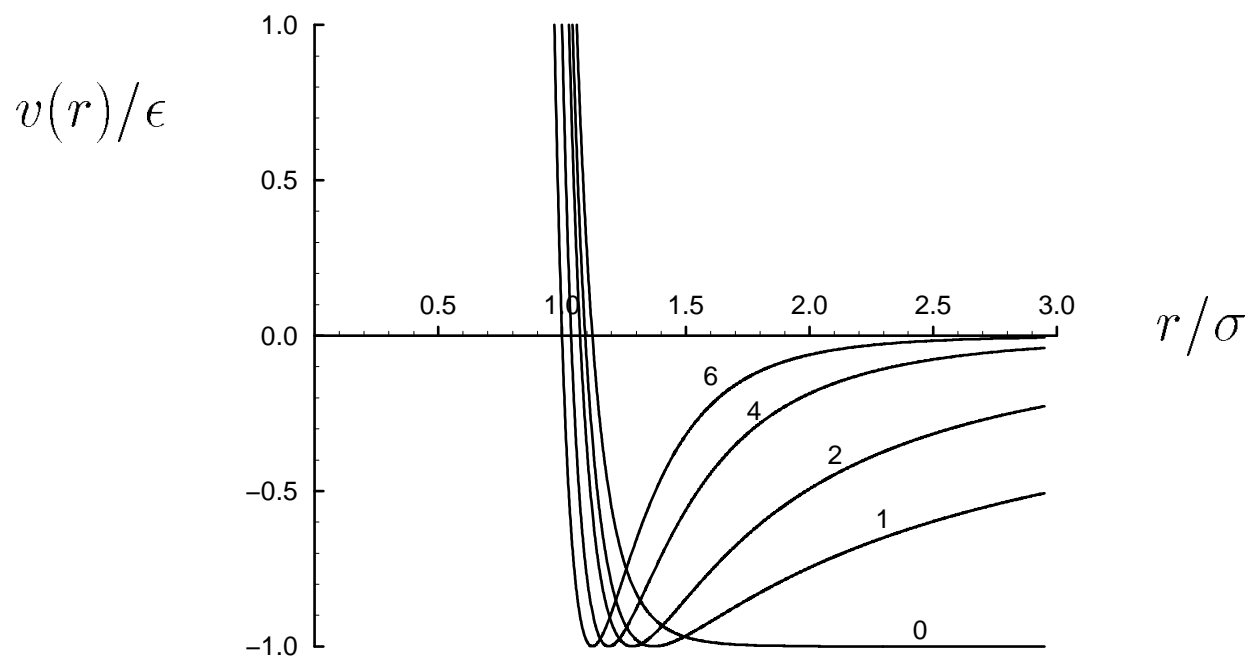

Figure 1 


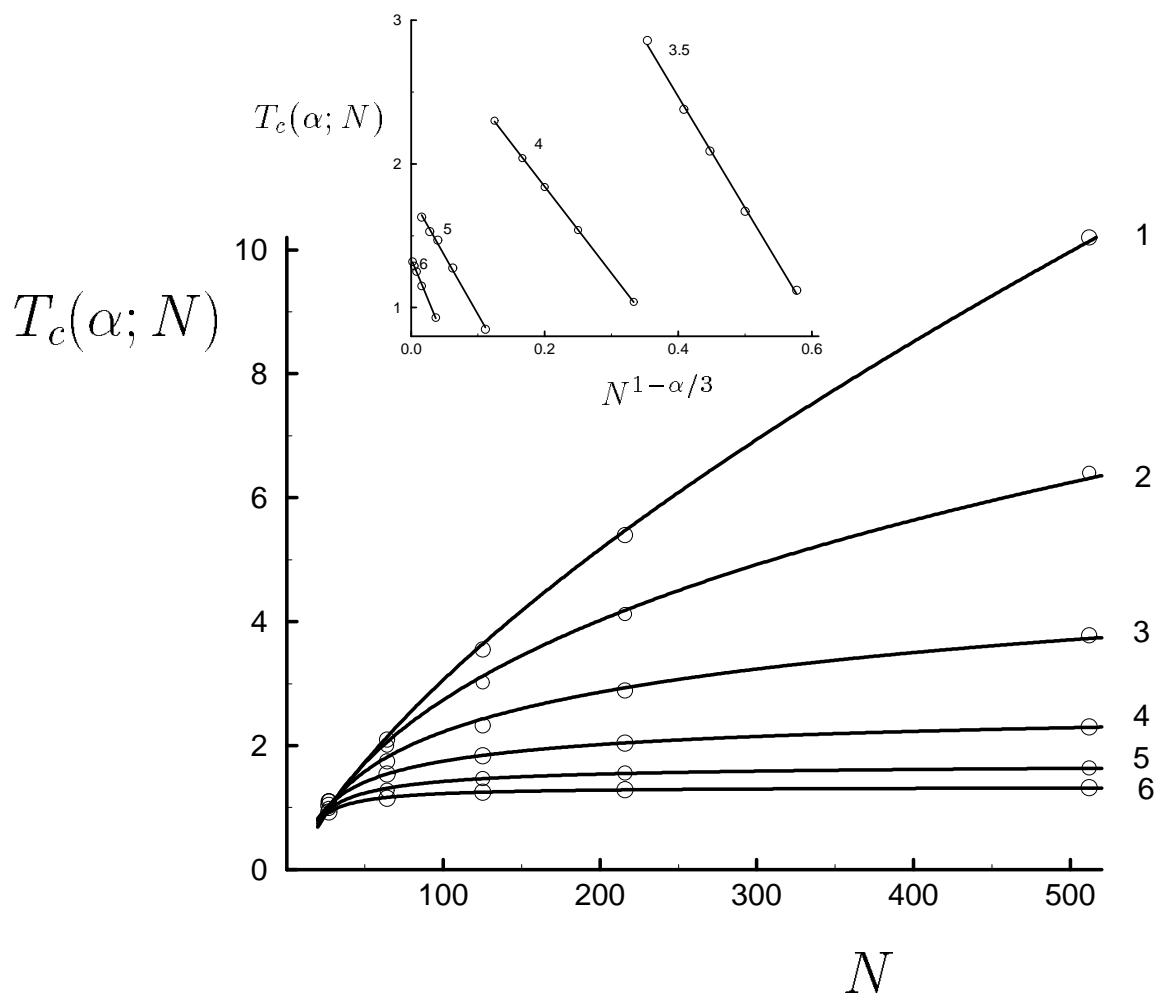

Figure 2 


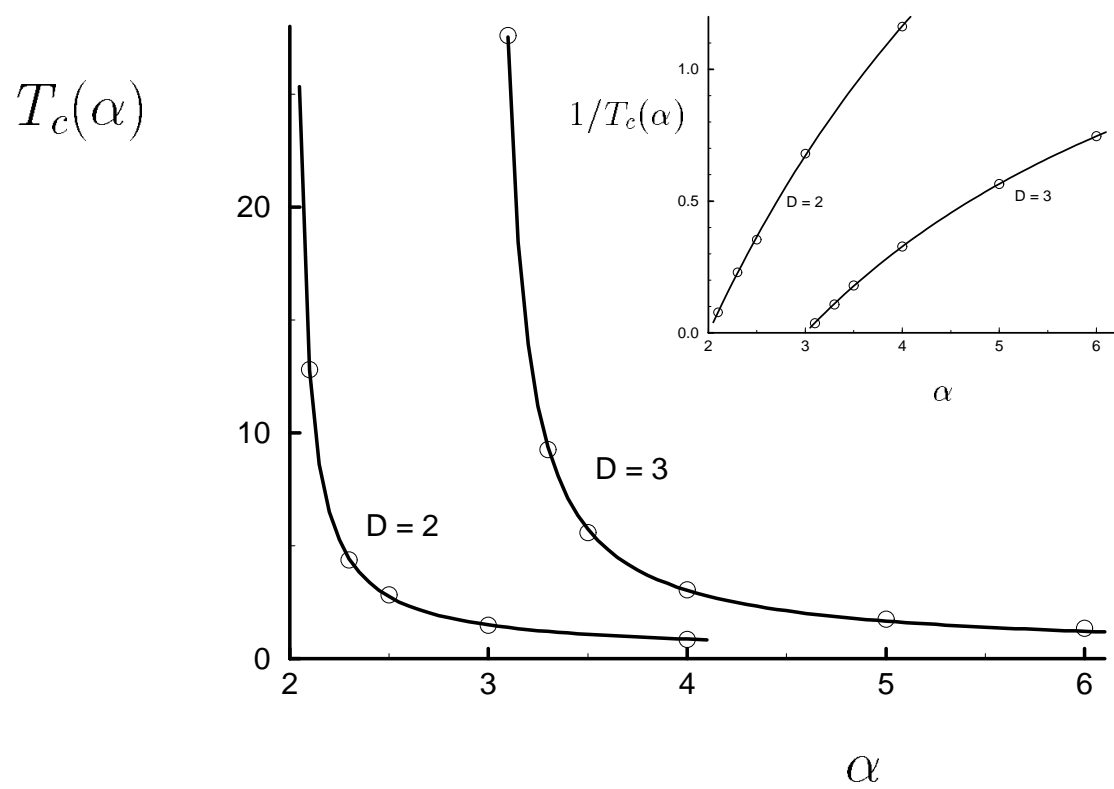

Figure 3 


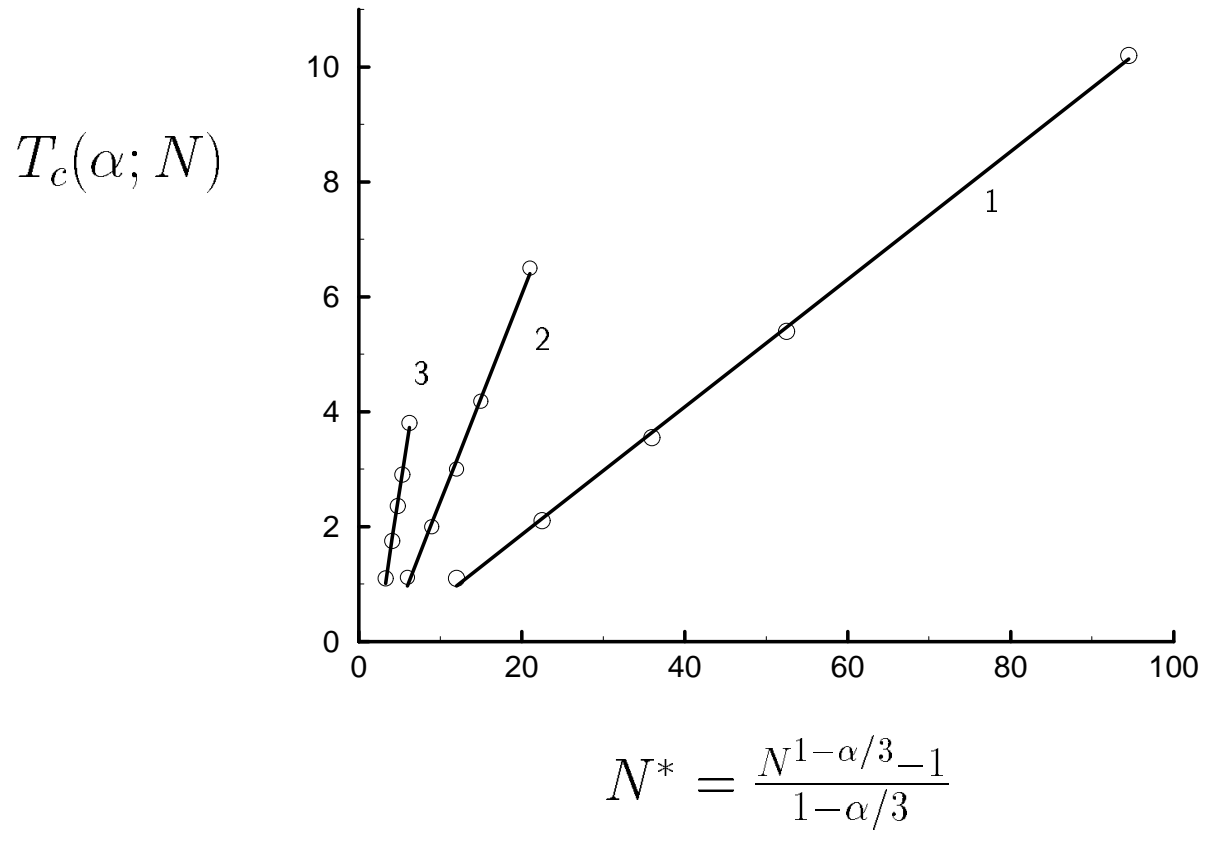

(a)

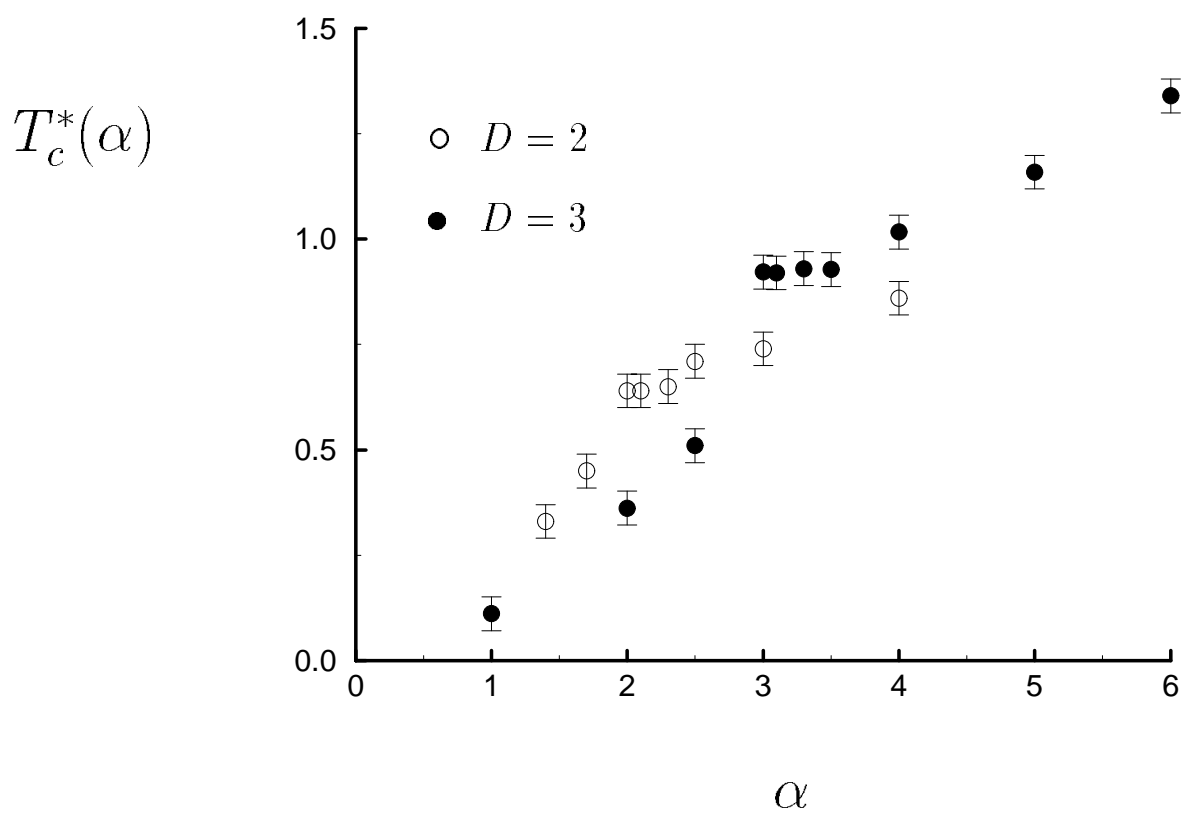

(b)

Figure 4 


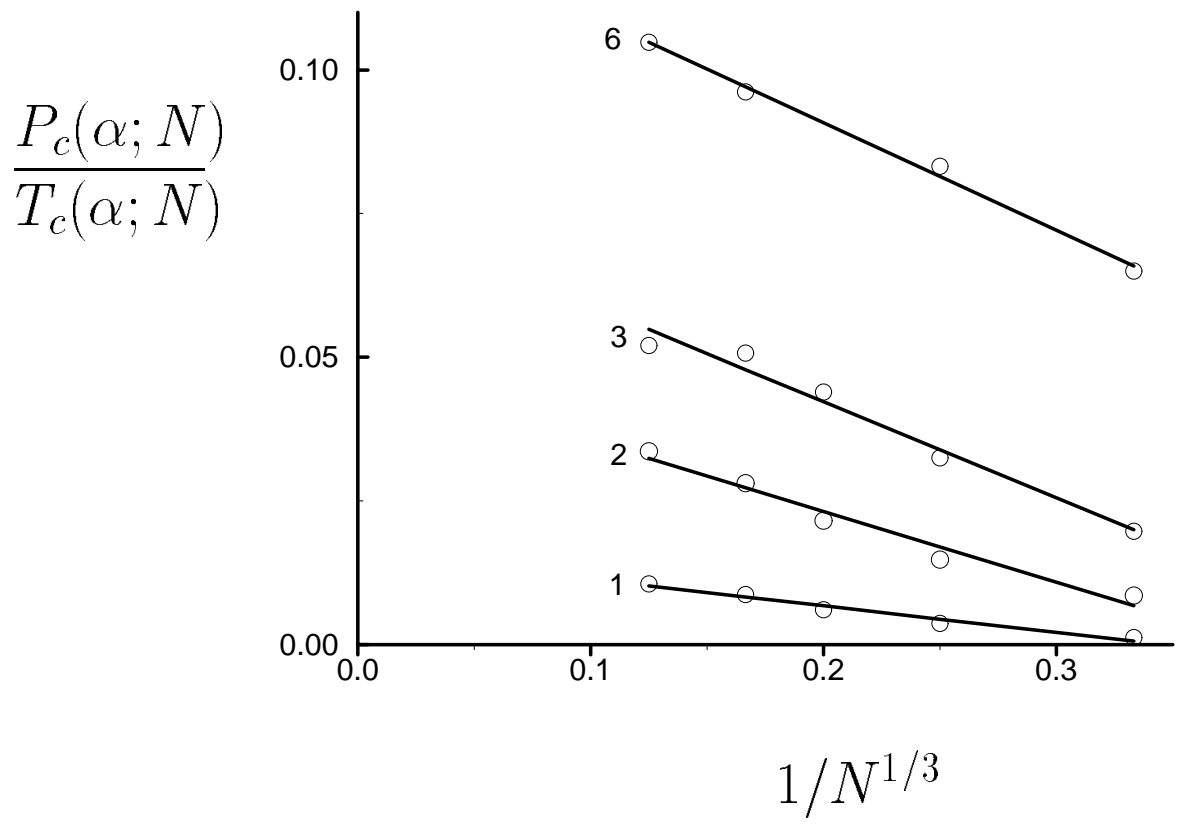

(a)

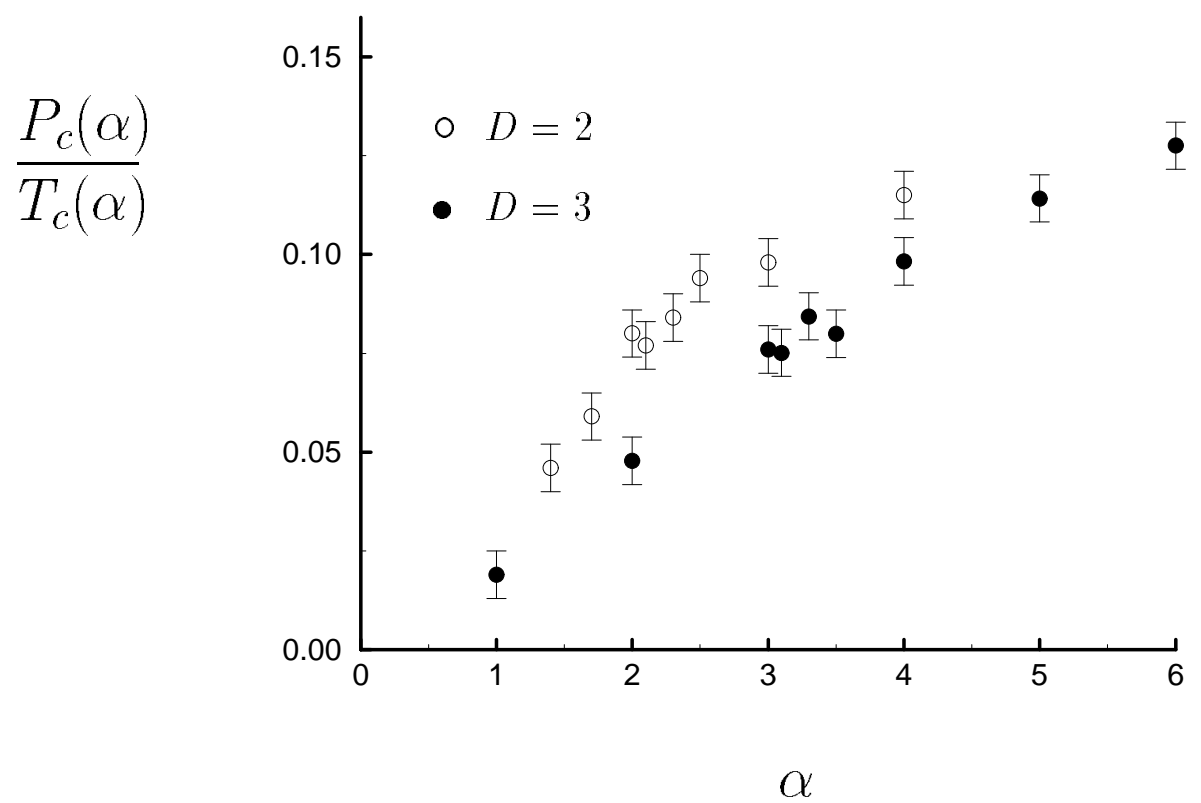

Figure 5 

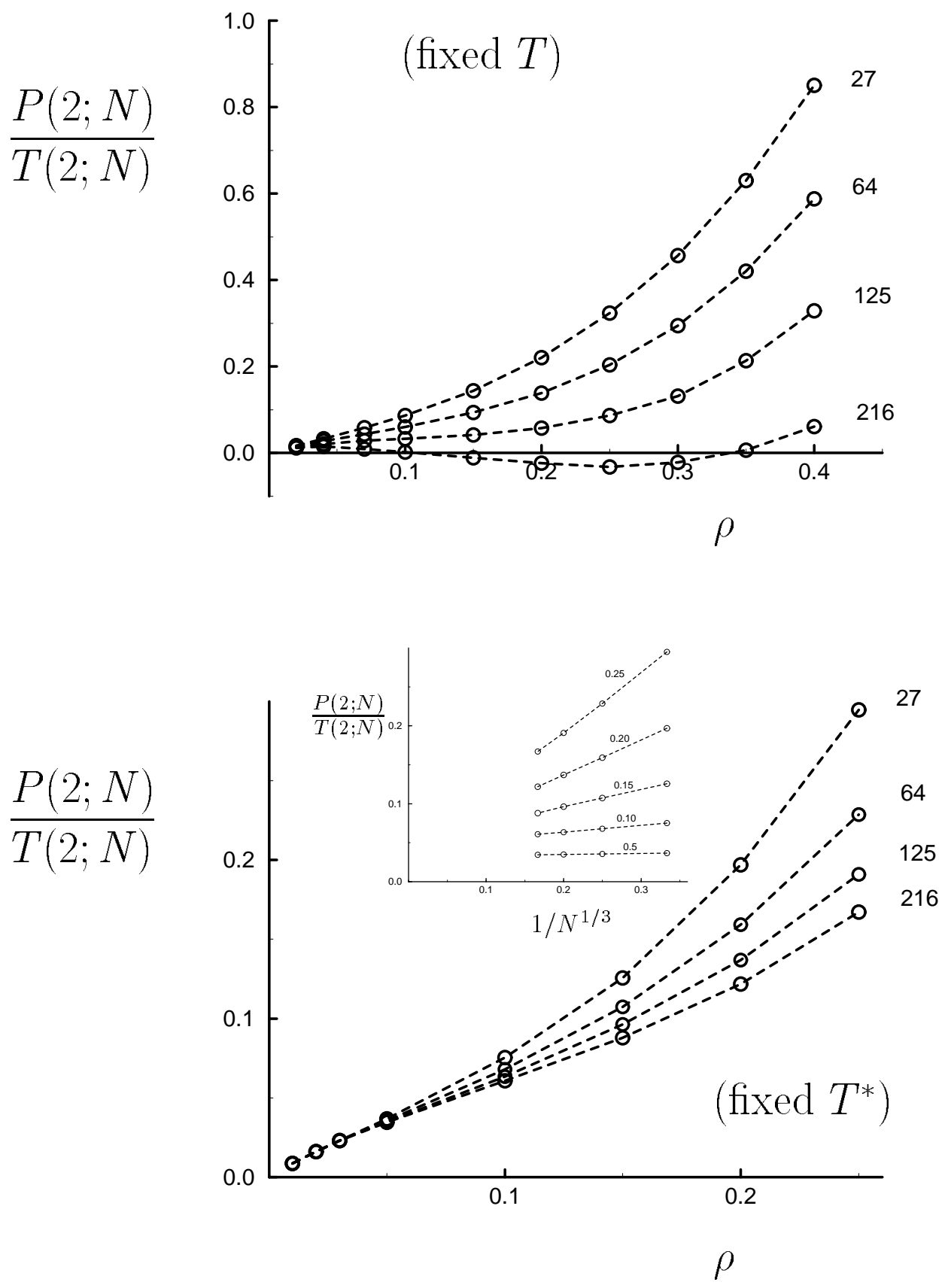

Figure 6 